\title{
Redistribution of Spatial Representation in the Hippocampus of Aged Rats Performing a Spatial Memory Task
}

\author{
Sheri J. Y. Mizumori, Annette M. Lavoie, and Anjali Kalyani \\ University of Utah
}

\begin{abstract}
Young and old rats performed on a maze according to a forced-choice and then a spatial memory procedure either in the same or a different environment. Aged rats were slower to learn the spatial memory task when tested in the same, but not in a different, room. One interpretation of this pattern of results is that although old rats learn new rules as quickly as young rats, they show less flexibility with old rules and familiar spatial information. Impaired choice accuracy during asymptote performance suggests poor processing of trial-unique information by old rats. Spatial correlates of hippocampal CA1 and hilar cells varied with task demand: CA1 cells of aged rats showed more spatially selective place fields, whereas hilar cells showed more diffuse location coding during spatial memory, and not forced-choice, tests. Such representational reorganization may reflect a compensatory response to age-related neurobiological changes in hippocampus.
\end{abstract}

Extensive literature indicates that normal aging is accompanied by a decline in the ability to acquire new information. Importantly, this impairment appears selective to certain types of information. For example, aged rats perform relatively poorly on complex visuospatial memory tasks (for a review, see Barnes, 1990). Past studies have ruled out the possibility that the impaired performance is due to changes in motor system function, motivation level, or visual function. The selectivity of the learning impairment has been demonstrated by many laboratories that found that aged rats perform as well as young rats on visually dependent, nonspatial maze tasks (Barnes, Green, Baldwin, \& Johnson, 1987; Lowry, Ingram, Olton, Waller, Reynolds, \& London, 1985; Rapp, Rosenberg, \& Gallagher, 1987; Winocur, 1988). Given that hippocampal lesions often produce deficits in tasks requiring animals to learn trial-unique data, the spatial learning impairment of old rats may be mediated by age-associated neurobiological changes in hippocampus. One could also argue that old rats have difficulty learning the rules associated with particular spatial tasks. The latter interpretation suggests the involvement of nonhippocampal brain structures (e.g., frontal cortex; Winocur \& Moscovitch, 1990). Therefore, we evaluated the relative contribution of rule-based and item- and event-based learning systems to the spatial navigation deficit shown by old rats. Also, given the extensive evidence indicating at least hippocampal involvement in the spatial decline of old animals (e.g., Barnes, 1979; Gallagher, Bostock, \& King, 1985), we investigated possible age changes in spatial coding by hippocampal neurons.

An important piece of evidence suggesting that hippocampus plays a special role in an organism's ability to navigate

Sheri J. Y. Mizumori, Annette M. Lavoie, and Anjali Kalyani, Department of Psychology, University of Utah.

This work was supported by National Institutes of Health Grant AG09299. We thank James G. Canfield for helpful discussions and Karen Burk and Leigh Hardy for assistance with behavioral testing and data analysis.

Correspondence concerning this article should be addressed to Sheri J. Y. Mizumori, Department of Psychology, 502 Behavioral Science Building, University of Utah, Salt Lake City, Utah 84112. effectively is the finding that "place cells" selectively discharge when animals occupy particular locations in their environment (Muller, Kubie, Bostock, Taube, \& Quirk, 1991; O'Keefe \& Dostrovsky, 1971). Although it has been suggested that place cells are related to the experience-dependent spatial navigation ability of rats (e.g., Mizumori, 1994; O'Keefe \& Speakman, 1987), it has yet to be demonstrated that age-related changes in place cell function contribute to the age-related change in spatial learning. Although Barnes, McNaughton, and O'Keefe (1983) reported that hippocampal place cells show less specific location coding in aged rats, the animals were not tested on a memory task.

To evaluate the specific contribution of hippocampus to age changes in spatial learning, young adult and aged rats were trained to perform first a nonspatial task and then a spatial task on a radial maze. To investigate the relative contribution of trial-unique and rule-based learning, we compared the rates of acquisition of a spatial navigation task when animals were trained in a room that was either the same or different from the one in which they learned a nonspatial task. If aged animals have more difficulty than young animals learning new rules, it was predicted that they would show slower rates of acquisition of the spatial memory task irrespective of the room being tested. Such a pattern of results could indicate frontal lobe dysfunction. Conversely, similar rates of acquisition between young and aged rats, together with a persistent increase in errors by aged rats during asymptotic performance, would implicate selective hippocampal involvement. According to past results, we expected to find behavioral evidence for at least hippocampal dysfunction. Therefore, an electrophysiological investigation of hippocampal place representation was conducted to ascertain whether age-related changes in spatial coding might contribute to the spatial learning deficit.

\section{Method}

\section{Animals}

Twenty-six Fischer-344 rats-13 young ( 9 months old) and 13 aged (24 months old) - were obtained from Harlan Sprague-Dawley via the 
National Institute on Aging. Free access to food and water was permitted for at least 10 days before the surgical implantation of recording electrodes. During that time, animals were weighed daily and handled. Lights were on in the colony room from 7 a.m. to 7 p.m. Behavioral testing occurred between 8 a.m. and 12 p.m.

\section{Electrode Construction and Surgical Procedures}

Hippocampal single-unit activity was monitored using the stereotrode recording method (McNaughton, O'Keefe, \& Barnes, 1983). Two lacquer-coated tungsten wires $(20 \mu \mathrm{m}$ diameter $)$ were twisted together, dipped in Epoxylite, and then baked. Each stereotrode was threaded through a 30-gauge stainless steel tube that was fastened to a miniature microdrive. Each microdrive contained two such tubes, and one microdrive was implanted over each hemisphere. The electrode tips were cut blunt and then gold plated to about $100 \mathrm{k} \Omega$ (tested at 1 $\mathrm{kHz}$ ).

For surgical implantation of the electrodes, the animals were initially anesthetized with $30 \mathrm{mg} / \mathrm{kg}$ of pentobarbital (Nembutal, 50 $\mathrm{mg} / \mathrm{ml}$ ) followed by supplements of $0.05 \mathrm{ml}$ as needed. Small burr holes were drilled in the skull at the following stereotaxic coordinates (Paxinos \& Watson, 1986): 4.0 to $5.0 \mathrm{~mm}$ posterior to bregma and \pm 2.4 $\mathrm{mm}$ lateral of the midsagittal suture. The stereotrodes were lowered about $1.5 \mathrm{~mm}$ below the dural surface at a site just dorsal to the hippocampus. A reference electrode $(114-\mu \mathrm{m}$ Teflon-coated stainless steel wire) was placed in the corpus callosum, and a ground lead was soldered to a jeweler's screw attached to the skull. All electrode and ground leads were inserted into a connecting socket that was permanently attached to the rat's head. The animals were allowed 7 days of recovery from surgery before commencement of behavioral analyses.

\section{Behavioral Testing Apparatus and Procedure}

Behavioral testing occurred on two eight-arm radial mazes (Mizumori \& Williams, 1993; Olton \& Samuelson, 1976). Briefly, each maze consists of eight alleys (or arms) that radiate from a round central platform. The center of each arm is hinged perpendicular to its length such that the proximal portion of the arm can be made flush with the central platform (to provide access to food reward located at the arm ends) or lowered (to restrict access to the arm ends). Experimenter control of the maze arms is accomplished using remote switches. The mazes are located in distinct rooms that contain several items that could serve as distal visual cues for solving the task (e.g., tables, chairs, and miscellaneous laboratory equipment). Each room is lit by a single $25-\mathrm{W}$ incandescent bulb.

Individual animals underwent the same sequence of behavioral tests. A summary of the experimental design can be found in Figure 1. During Phase 1 of training, all animals performed on the maze according to a forced-choice procedure. Half of the subjects in each age group were trained in Room 1 (Groups 1 and 3), and the remaining animals were trained in Room 2 (Groups 2 and 4). During Phase 2, the same animals learned a spatial memory task on the maze. Half of the animals remained in the same room during both Phases 1 and 2 (Groups 1 and 2), whereas the other animals underwent spatial memory training in a room different from that used for forced-choice training (Groups 3 and 4). This design was used so that it would be possible to examine age-related changes in the rates of acquisition of a new spatial task when animals were required to use familiar or new spatial information.

The preliminary training portion of Phase 1 involved first placing the rat on the central platform with access to a single maze arm. Chocolate milk reward was placed at the arm end. The particular arm selected varied randomly across days of preliminary training. When rats readily

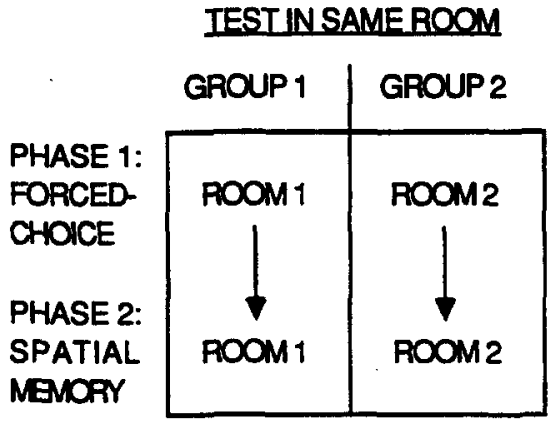

\section{TESTINDIFFERENT ROOMS}

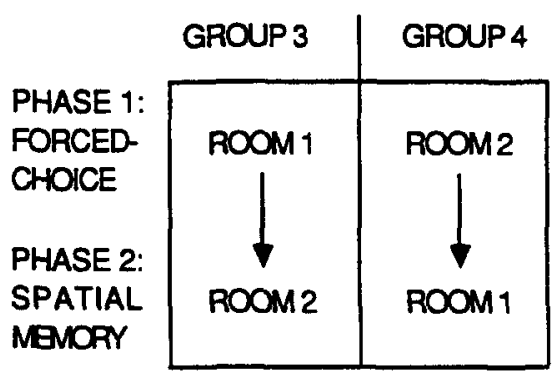

Figure 1. Summary of the experimental design for the behavioral testing of animals. All rats started maze training according to the forced-choice procedure (Phase 1; see text for explanation). Each group was composed of both young adult and aged rats. Groups 1 and 3 received forced-choice training in Room 1, whereas Groups 2 and 4 received training in Room 2 . After completion of Phase 1 , the same animals underwent spatial memory training (Phase 2; see text for explanation) either in the same room as forced-choice training (Groups 1 and 2) or in a room that was different from the one used in forced-choice training (Groups 3 and 4).

traversed the single maze arm to obtain the reward, forced-choice maze training began. During this period, the rats were required to enter each of eight maze arms that were individually and sequentially presented. The order of the arms presented was randomly determined without replacement. Traversals of all eight arms constituted a single trial. Such training continued until eight trials were completed in 1 hour (2-minute intertrial interval) for 7 consecutive days. On achievement of criterion performance, the electrodes were slowly lowered into the hippocampus. When new, well-isolated units were encountered, the behavioral correlates of cell discharge were analyzed. Otherwise, the rats were run on the maze at least every other day to maintain asymptote performance levels. In this way, all behavioral analyses of cellular activity were recorded during peak performance.

Forced-choice testing continued until the recording electrodes were lowered through the hippocampus of the first hemisphere (about $4 \mathrm{~mm}$ ventral to the dural surface). At that time, animals began Phase 2 of maze training according to a spatial memory procedure (Mizumori, Perez, Alvarado, Barnes, \& McNaughton, 1990). At the beginning of each spatial memory trial, four randomly selected arms were presented to the rat in sequence. After the rat had traversed these four arms, all eight maze arms were presented simultaneously. The rat was required to retrieve the remaining four rewards by not entering the four arms previously entered that trial. Re-entries were counted as errors. Criterion performance was achieved when the rats completed eight trials per day (two-minute intertrial interval) for 14 consecutive days. At this time, the recording electrodes were lowered toward the 
hippocampus of the second hemisphere. The rats performed the spatial task every other day unless well-isolated units were encountered, in which case animals ran the maze daily. As in the case of forced-choice testing, all hippocampal cellular activity was recorded during asymptotic maze performance.

\section{Single Unit and Behavioral Recording Procedures}

The stereotrode recording method (McNaughton, O'Keefe, \& Barnes, 1983) involves the simultaneous and independent recording of neuronal activity on each wire of an electrode pair. Incoming signals were amplified 4,000 to 10,000 times and then filtered (at half amplitude) between $600 \mathrm{~Hz}$ and $6 \mathrm{kHz}$. The signals were then passed through a window discriminator such that a 1-ms sampling period began when a signal from either channel surpassed a predetermined threshold. The entire waveform was collected by a BrainWave Neuroscience Workstation AST 80386-based computer system (sampling frequency of $32 \mathrm{kHz}$ ). This system allowed the experimenter to isolate single units with the aid of a cluster analysis routine. The system software processed the signals according to at least eight parameters (four for each recording channel): the maximum and minimum voltages of the sampled waveform, and the latencies of these values from the onset of the sampling period. Also a template-matching algorithm was used to further separate unique waveform shapes that corresponded to identified clusters occupying a given parametric space. After determination of each cluster's parametric space, it was subsequently analyzed for its behavioral correlates.

For all recording sessions, the connecting socket on the rat's head was attached to a headstage that contained five unity gain preamplifiers and an infrared light-emitting diode (LED). The rat's movements about the maze were monitored by an automatic tracking system that sampled the $\mathrm{XY}$ coordinates of the diode at a frequency of about 20 $\mathrm{Hz}$. The time of each position sample and spike event was also logged.

\section{Unit Identification and Analysis}

Hippocampal neuronal activity can be distinguished according to one of two patterns of spike discharge. Complex-spike (CS) cells discharge high-frequency bursts, usually with progressively declining spike amplitudes within a burst. These cells are encountered near the CA1 pyramidal cell layer as well as within the dentate gyrus hilar region (Fox \& Ranck, 1975; Ranck, 1973). Single-spike (SS) cells, alternatively, discharge single-action potentials with comparatively long interspike intervals. SS cells are typically encountered in stratum oriens, in stratum pyramidale, and near stratum granulosum (Fox \& Ranck, 1975; Mizumori, McNaughton, Barnes, \& Fox, 1989; Rose, Diamond, \& Lynch, 1983). CA1 neurons were identified as such if they were encountered about $2 \mathrm{~mm}$ below the dural surface. Other CS and SS neurons were classified as hilar cells because they were encountered about 700 to $900 \mu \mathrm{m}$ ventral to CA1. The categorization of cells as CS or SS neurons was later verified by visual inspection of autocorrelation functions (Mizumori et al., 1989). The location of the electrode track was verified with standard histological techniques. Frozen sections ( $40 \mu \mathrm{m}$ thick) were stained with cresyl violet and then observed under a light microscope.

The behavioral correlate of a majority of CS neurons is locationspecific firing (O'Keefe \& Dostrovsky, 1971; McNaughton, Barnes, \& O'Keefe, 1983; Mizumori et al., 1989; O'Keefe, 1976), whereas the primary behavioral correlate of SS neurons in the hippocampus is voluntary movements of the animal through its environment (Mizumori, Barnes, \& McNaughton, 1990, 1992; Ranck, 1973). Thus, given that a primary interest of this study was age changes in spatial coding by hippocampal cells, the specificity of spatial coding by the CS neurons was analyzed as follows. The firing rates as the rat moved inward and outward on the eight maze arms were calculated. The highest of these 16 rates was divided by the average of the remaining 15 rates to arrive at a spatial specificity score. It is important to note that our calculations incorporate not only the location specificity component of place fields but also the directional component. Thus, a cell that discharged when a rat moved inward on one maze arm was assigned a higher spatial specificity score than a cell that showed the same size place field but fired as the rat moved both inward and outward on the maze arm containing the place field. Also it is worth pointing out that this algorithm for determining spatial specificity will tend to underestimate true spatial selectivity because most place fields cover an area that is smaller than that of one maze arm. Nevertheless, this method of quantification is sufficiently sensitive to experimental manipulations that important properties of place fields have been revealed in past experiments (e.g., McNaughton, Barnes, \& O'Keefe, 1983; Mizumori et al., 1989).

The reliability with which a cell exhibited the same location-specific firing across trials was quantified by calculating a reliability index. Specifically, this index reflects the proportion of trials in which the highest of the 16 firing rates (described previously) was associated with a particular arm of the maze.

A third measure of spatial representation was quantified to obtain a measure of the directional selectivity with which place cells fired. The average rate in the inward direction of each arm was compared with the average rate on the outward direction on opposite maze arms to arrive at a mean rate of cell discharge as an animal moved along eight possible trajectories defined by the maze. The resultant magnitude of the vector sum is referred to as the mean vector length (MVL). MVL values ranged from 0 to 1 ; an MVL of 1 reflects a cell that fired only as the animal moved either inward or outward through a cell's place field.

Finally, Pearson $r$ correlation analyses were performed to examine the relationships among specificity, reliability, and MVL measures of place field properties. For all three measures, larger values are considered more desirable for meaningful and precise spatial coding. Therefore, it was of interest first to determine the relationships between these measures in young animals and then evaluate possible age or task influences on these relationships.

Graphically, the location specificity of cell discharge was illustrated by generating "spot-rate" plots. The mean firing rate of the cell was determined while the rat remained within a five-pixel radius $(7.5 \mathrm{~cm})$ of the first position sampled. When the diode moved outside this radius, the first new position point served as the next integration center, and the firing rate that corresponded to that location was determined. The spot-rate graphs consists of dots (indicating occupied positions on the maze) and circles whose radii were linearly proportional to the local firing rate of the cell. Vectors radiating from the center of the circles indicated the direction of diode movement when the rat occupied that particular location on the maze.

\section{Results}

\section{Behavioral Analysis}

After preliminary training with the forced-choice procedure, young and old animals required a similar number of days to achieve criterion performance on the forced-choice task $(p>.10)$. All unit recording sessions occurred during asymptote performance. The mean $( \pm \mathrm{SE})$ time required to complete maze trials when rats performed at asymptote was significantly different between age groups irrespective of the particular room in which animals were tested, $F(1,157)=5.4, p<.05$. Aged rats required $226 \pm 30.0 \mathrm{~s}$ to complete forced-choice trials, whereas young rats completed such trials in $158 \pm 9.2 \mathrm{~s}$ (Figure 2). That old rats require more time to perform tasks 


\section{FORCED CHOICE}

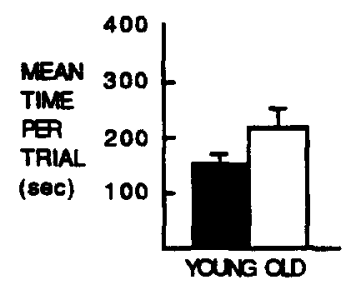

\section{SPATIAL MEMORY}

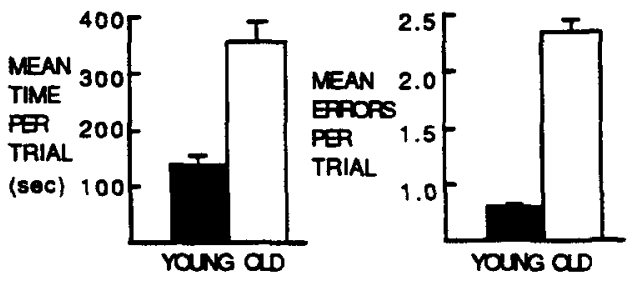

Figure 2. Top: The mean time required to complete asymptotic forced-choice trials on the radial maze. As found in other investigations, aged rats required significantly more time to finish maze trials than young rats. Bottom, left panel: Aged rats also required more time to complete asymptotic spatial memory trials than young rats; right panel: aged rats made significantly more errors than young rats.

has been shown in a variety of behavioral situations (for a review, see Barnes, 1990).

The mean number of errors made during acquisition of the spatial memory task (Phase 2) was evaluated in different ways. For each age group of animals that remained in the same room for both forced-choice and spatial memory tests, there was no significant effect $(p>.10)$ of room on the rate of acquisition of the task. Therefore, data from animals that remained in the same room for both phases of training were pooled for subsequent analyses (same room condition: Groups 1 and 2). Within each age group, there were no significant differences $(p>.10)$ between animals that were forced-choice trained in Room 1 and then switched to Room 2 for spatial memory training and those animals forced-choice trained in Room 2 and then switched to Room 1 for spatial memory training. Therefore, these animals were considered as a single group for subsequent analyses (different room condition: Groups 3 and 4).

The rates of acquisition of the spatial memory task were compared between young and aged rats for the same and different room conditions. Pilot data indicated that both age groups of rats, when first trained according to the forcedchoice procedure, achieved asymptote performance on the spatial task within 14 days. Therefore, the mean number of errors committed during the first 14 days of spatial memory training was compared between young and aged rats in the same room condition (Figure 3, top) and in the different room condition (Figure 3, bottom). With regard to the subjects in the same room condition, a repeated measures ANOVA revealed that both aged and young rats showed significant improvement over days of training, $F(1,13)=23.61, p<.01$. Although there was no main effect of age, $F(1,13)=2.58, p>.10$, a significant interaction effect of age and repeated measures was found,
$F(1,13)=2.28, p<.01$. Thus, although both young and old rats showed significant learning in this task, the statistical interaction indicates that the old rats acquired the memory task at a slower rate than young rats trained in the same room in which they received forced-choice testing.

When the rats learned the spatial memory task in a room that was different from the forced-choice testing room (different room condition), both young and old rats showed significant improvement across trials, $F(1,13)=15.70, p<.01$. Also a significant age effect, $F(1,9)=13.80, p<.01$, indicated that, overall, the aged rats made more errors than young rats. Unlike animals in the same room condition, however, a significant interaction effect of age and repeated measures was not observed, $F(1,13)=0.49, p>.10$. Thus, when young and aged animals learned the spatial task in a different room, both showed significant and similar rates of improvement in performance over time, and aged rats acquired the task at the same rate as young animals, even though they exhibited less accurate performance on the spatial maze overall.

During asymptotic performance on the spatial memory task, old rats of Groups 1 and 2 made significantly more errors than comparable young rats (old: $1.76 \pm 0.25$; young: $.59 \pm 0.10$ ), $F(1,142)=20.53, p<.001$ (see Figure 2). Also the old rats required more time on the maze to complete a trial than young rats (old: $359.7 \pm 30.9 \mathrm{~s}$; young: $148.8 \pm 10.9 \mathrm{~s}$ ), $F(1,142)=$ $51.26, p<.001$.

\section{Single-Unit Analysis}

Of the 617 cells recorded from both young and old rats, 420 were classified as CS cells and 197 were considered SS cells. Because the purpose of this study was to examine age-related

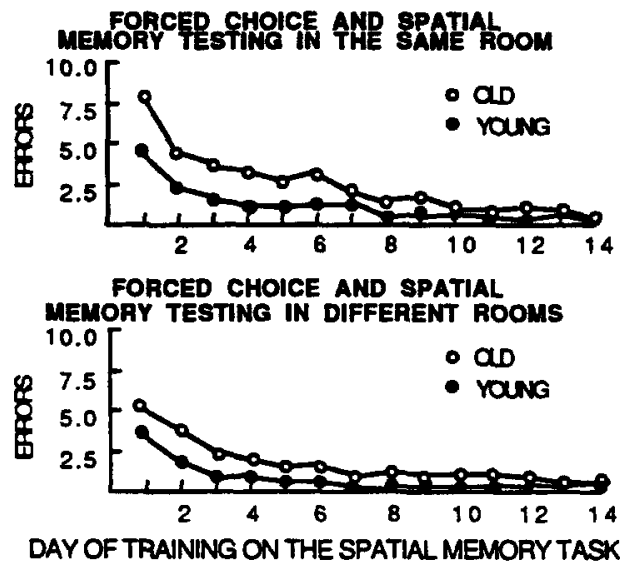

Figure 3. Mean number of errors made during the first 14 days of acquisition of the spatial memory task. The data were divided according to whether forced-choice and spatial memory training occurred in the same (top) or different (bottom) rooms. When tested in a familiar room, aged rats learned the spatial task at a slower rate than young rats. In contrast, no significant age difference in learning rate was observed when animals learned the spatial task in a novel spatial environment despite the fact that aged rats generally made more errors than young rats. The overall pattern of behavioral effects indicates that, when compared with young rats, aged rats may have more difficulty using familiar spatial information in different ways. 
changes in the spatial properties of hippocampal cells, and place representation is a more prominent characteristic of CS cells than SS cells (e.g., McNaughton, Barnes, \& O'Keefe, 1983; Mizumori et al., 1989; Muller et al., 1991; Ranck, 1973), age effects on place cell properties are reported for only the CS neurons. There were no age differences in mean spike amplitude, spike duration, and mean firing rates of CA1 or hilar CS neurons-young CA1 $(n=148)$ : amplitude, $165.5 \pm 9.0 \mu \mathrm{V}$; duration, $313.5 \pm 6.0 \mu \mathrm{s}$; rate, $1.36 \pm 0.25 \mathrm{~Hz}$; old CA1 ( $n=117)$ : amplitude, $178.9 \pm 13.19 \mu \mathrm{V}$; duration, $299.2 \pm 6.1$ $\mu$; rate, $1.26 \pm 0.39 \mathrm{~Hz}$; young hilar $(n=75)$ : amplitude, $191.9 \pm 17.9 \mu \mathrm{V}$; duration, $290.1 \pm 6.4 \mu \mathrm{sec}$; rate, $.54 \pm 0.18$ $\mathrm{Hz}$; old hilar $(n=80)$ : amplitude, $186.8 \pm 12.6 \mu \mathrm{V}$; duration, $285.0 \pm 6.7 \mu \mathrm{s} ;$ rate, $1.72 \pm 1.32 \mathrm{~Hz}$.

\section{Spatial Correlates of CS Neurons}

There was no statistical effect of room on place specificity, reliability, or MVL measures of CS cells recorded from animals trained according to the forced-choice procedure. Thus, cells recorded in the two rooms showed similar spatial properties. There were also no significant differences between Room 1 and 2 in spatial measures for cells recorded as rats performed the spatial memory task. Furthermore, place specificity, reliability, and MVL measures did not differ for animals that received forced-choice and spatial memory training in the same or different rooms. Consequently, the data were pooled across rooms such that all forced-choice trial data were compared with all spatial memory trial data. Separate threeway analyses of variance (ANOVAs) were performed on each of the place specificity, reliability, and MVL scores. The contrasting factors of these ANOVAs were age (young, old), task (forced-choice, spatial memory), and cell region recorded (CA1, hilus). Three-way interaction effects were observed for place specificity, $F(1,412)=6.92, p<.01$, and MVL measures, $F(1,412)=5.02, p<.05$. These statistical results suggest that any age effects on place specificity or MVL depended on the task being performed and the cell region being recorded. No such interaction effect was observed for reliability scores. A more detailed analysis of these interaction effects is presented next.

Figure 4 (top) compares the spatial specificity index of young and aged rats trained according to the forced-choice procedure. The values for CA1 (young $n=74 ;$ old $n=68$ ) and hilar (young $n=29$; old $n=33$ ) neurons are shown in the left and right panels, respectively. One-way ANOVAs revealed no significant age effects for either cell type, indicating that both populations of cells coded location information with similar degrees of specificity $(p s>.10)$. Also similar proportions of CA1 and hilar CS cells showed location-selective discharge (i.e., had specificity scores greater than 2.0; Lavoie \& Mizumori, 1994; Mizumori, Ward \& Lavoie, 1992): $81.1 \%$ and $83.8 \%$ of young and old CA1 CS cells, respectively, had place fields, and $89.7 \%$ of young and $87.9 \%$ of old hilar CS cells showed localized discharge during forced-choice tests.

There was no age effect on reliability (Figure 5 top). However, the MVL for CA1 cells of the old rats was greater than that observed for young rats, $F(1,140)=4.41, p<.05$ (Figure 6 top), an effect that hilar neurons did not show.

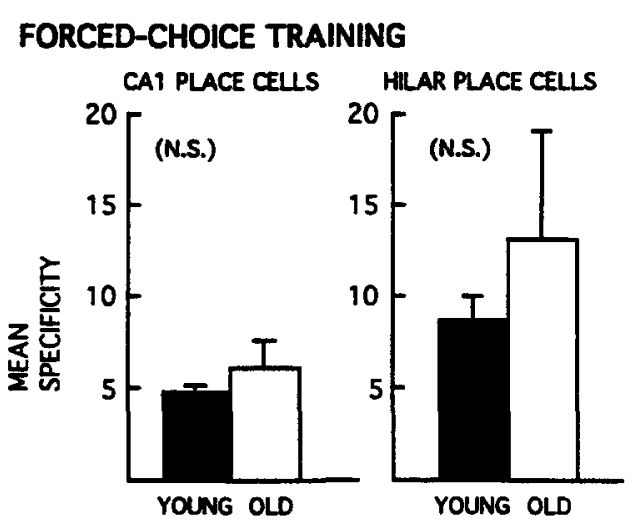

\section{SPATIAL MEMORY TRAINING}

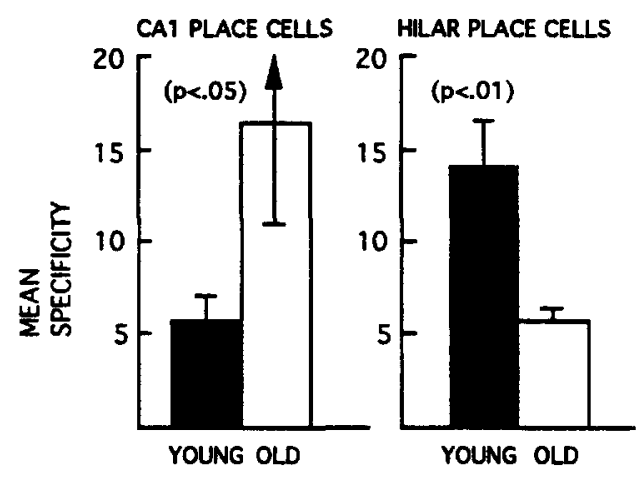

Figure 4. The mean $( \pm \mathrm{SE})$ place specificity scores for place cells recorded in the CA1 and hilar regions of the hippocampus as a function of age of the animal. Greater specificity scores indicate more localized place fields. Top: During asymptotic performance on the forced-choice task, place fields of young and aged rats are compared in terms of place specificity scores. For both age groups, hilar neurons show more specific place fields than CA1 neurons. No age differences were observed for these two cell types. Bottom: In contrast, a significant interaction effect was observed for cell type and age when rats performed the spatial memory task: whereas young rats showed the typical pattern of hilar cells exhibiting more specific place fields than CA1 cells, the CA1 cells of aged rats showed greater specificity than hilar cells. Furthermore, the CA1 place cells of aged rats were more specific than similar cells recorded from young rats, and the hilar place fields of aged rats were less specific than similar cells from young rats. Thus, there appears to be a task-dependent change in the organization of spatial representation in the aged hippocampus.

Graphic illustrations of representative place fields recorded during forced-choice testing for CA1 and hilar neurons are shown in Figure 7.

In contrast to what was observed during forced-choice training, the CA1 place fields recorded while aged rats $(n=49$ cells) performed the spatial memory task exhibited significantly greater specificity than similar cells recorded from young animals ( $n=74$ cells) performing the spatial memory task, $F(1,121)=4.81, p<.05$ (Figure 4 , bottom left). This was observed even though a similar proportion of young and old CA1 CS cells had place fields: $87.8 \%$ of young and $91.8 \%$ of old CA1 CS cells showed place-specific firing. There was no significant age effect on the reliability with which place fields were exhibited (Figure 5, bottom left). However, a significant 
FORCED-CHOICE TRAINING

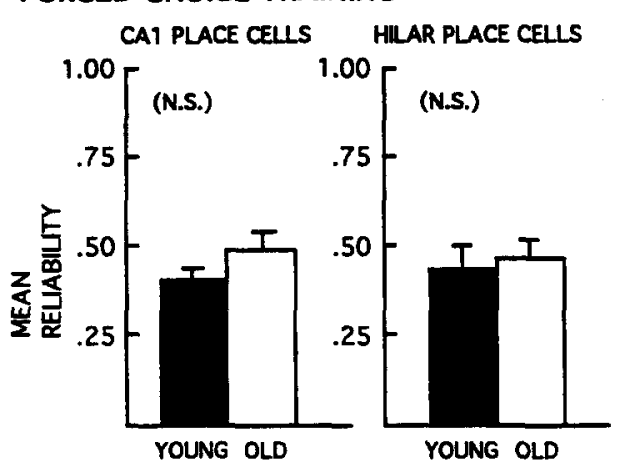

SPATIAL MEMORY TRAINING

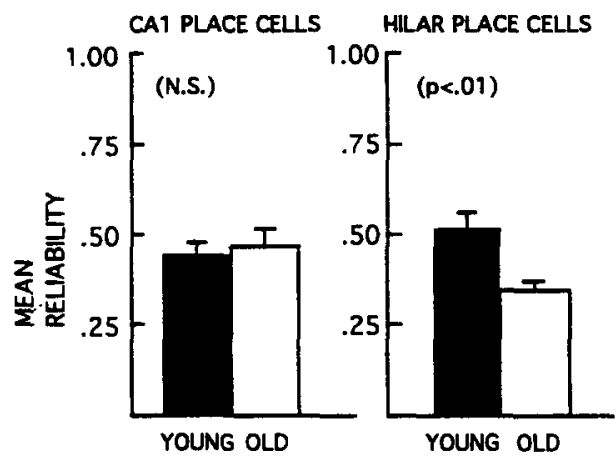

Figure 5. The mean $( \pm \mathrm{SE})$ reliability scores for place cells recorded in the CA1 and hilar regions of hippocampus as a function of age of the animal. Cells with a reliability score of 1.0 always fired when the rat entered the location of the place field. Top: During asymptotic performance on the forced-choice task, there were no differences in place field reliability as a function of area of hippocampus recorded or age of the animal. Bottom: When animals performed the spatial memory task, the reliability measure did not differentiate CA1 from hilar place fields. In contrast, the reliability score for hilar place cells recorded from aged rats was significantly lower than that of young animals. These data, together with those of Figure 4, reveal that hilar neurons lose both specificity and reliability in old age.

age effect was observed with aged rats showing greater directional specificity of discharge (as indicated by the MVL measure), $F(1,121)=10.82, p<.01$ (Figure 6, bottom left). It appears, then, that the CA1 place cells of old rats performing a spatial memory task were more specific and directionally tuned than those of young rats.

Hilar place fields, alternatively, were overall significantly less specific for old rats than young rats when performing at asymptote on the spatial memory task, $F(1,91)=9.77, p<$ 0.01 . Again, similar proportions of hilar CS cells showed place fields for young and old rats: $89.1 \%$ of young and $93.6 \%$ of old hilar CS cells showed place fields. In addition, the reliability with which the hilar place fields were exhibited across trials was lower in old animals, $F(1,91)=6.95, p<0.01$, and the directional bias of place cell discharge (i.e., MVL score) was reduced with age, $F(1,91)=9.75, p<0.01$.

In an attempt to determine whether these changes in place field properties were correlated with choice accuracy, Pearson $r$ correlations were calculated comparing individually the specificity, reliability, and MVL measures of CA1 and hilar CS cells with the average number of errors committed per trial during each recording session involving spatial memory training. None of the correlation coefficients were found to be statistically significant for either young or old rats. When young and old rat data were combined, however, a significant positive relationship was found between choice accuracy and spatial specificity scores of hilar neurons $(r=.25, p<.02)$ but not CA1 CS cells $(r=.01)$. It can be seen from Figure 8 that the distribution of scores was different between age groups: Young rats made generally fewer errors and had hilar cells with rather high spatial selectivity, and old rats tended to make more errors and their hilar neurons generally had lower specificity scores.

The relationships between location specificity and reliability or MVL measures were also examined. Positive statistical

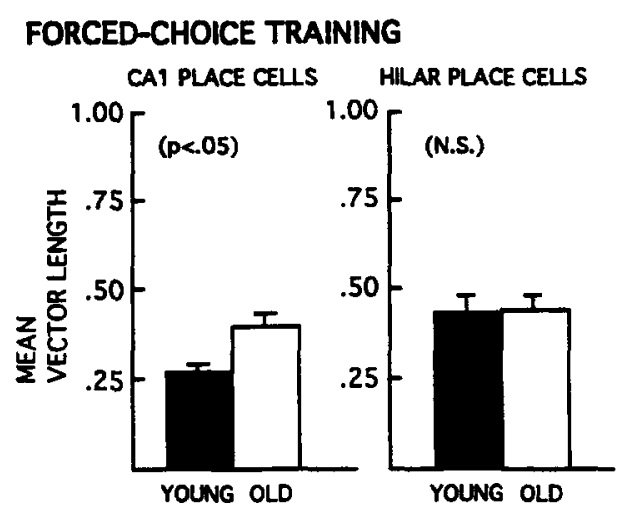

SPATIAL MEMORY TRAINING

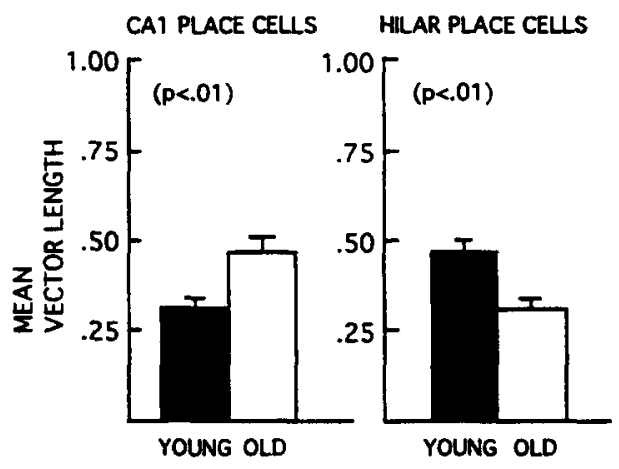

Figure 6. The mean $( \pm \mathrm{SE})$ vector length scores for place cells recorded in the CA1 and hilar regions of the hippocampus as a function of the age of the animal. CA1 place cells of aged rats showed more directional firing within place fields during both forced-choice and spatial memory tests. Hilar neurons of aged rats, on the other hand, exhibited a significant reduction in directional firing only during the spatial memory tests. Summarizing the data from Figures 4 to 6 , the most consistent age-related change in hippocampal place fields relates to those of both $\mathrm{CA} 1$ and hilar neurons when animals perform the spatial memory task: CA1 cells show elevated place specificity and directional firing, whereas hilar neurons exhibit less specific, less reliable, and less directionally selective firing. In contrast, the only significant age difference observed in place cell characteristics during the forced-choice task is enhanced directionality within the place fields of CA1 neurons. 
FORCED-CHOICE TRAINING CA1 PLACE CELLS

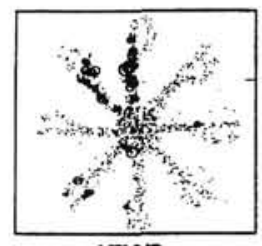

YONG

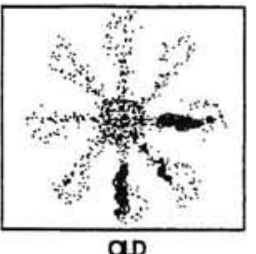

aD

SPATIAL MEMORY TRAINING CA1 PLACE CELLS
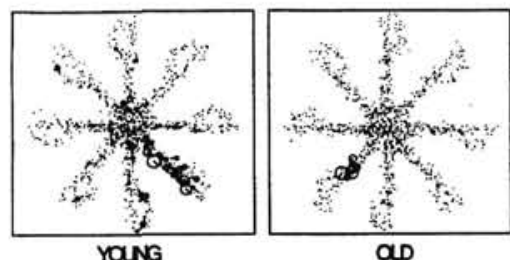

$\alpha$

FORCED-CHOICE TRAINING

HILAR PLACE CELLS

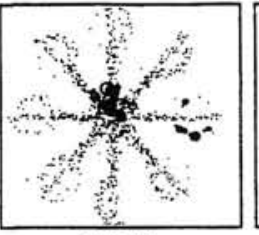

YONG

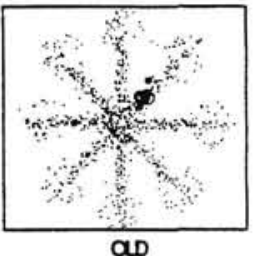

SPATIAL MEMORY TRAINING HILAR PLACE CELLS

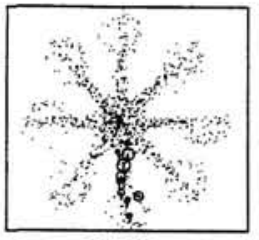

YoNG

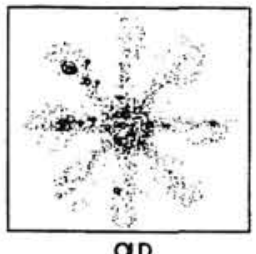

ap

Figure 7. Illustrations of place fields recorded from CA1 (left panels) and hilar (right panels) neurons of young and old rats during the forced-choice (top panels) and spatial memory tasks (bottom panels). Dots represent the locations occupied by the rat during multiple maze trials. Circles indicate the location of the rat when the cell fired. The size of the circles is proportional to the local firing rate, and vectors radiating from the center of the circle show the direction of movement when the cell fired. In the top left panels, it can be seen that during forced-choice testing the size of the CAl place fields of young and aged rats is similar. The cell recorded from a young animal preferentially fired when the rat moved outward on the north and northwest arms of the maze, whereas the cell recorded from the aged rat showed firing localized primarily to the east and south arms of the maze. The same age relationship is true for the examples of hilar place cells (top right). No differences in place specificity were observed. In contrast, the CA1 and hilar place cells recorded during the spatial memory trials showed significant age differences in specificity. The bottom left panels show a more specific place field recorded from the aged rat, whereas the bottom right panels demonstrate that the least specific field was recorded from an aged rat.

correlations were found between specificity and reliability measures as well as for specificity and MVL measures of CA1 and hilar place fields recorded from young rats performing either the forced-choice or spatial memory tasks (Tables 1 and 2). Thus, smaller place fields tended to also be more reliable and more directionally selective. In contrast to this pattern for young rats, place field properties of cells recorded from aged rats showed a task-dependent relationship: During forcedchoice training, significant correlations were observed between specificity and reliability measures for hilar, but not for CA1, CS cells. During spatial memory testing, in contrast, significant correlations were observed between specificity and reliability as well as between specificity and MVL for both CA1 and hilar populations of CS cells. The latter pattern is the same as that observed in young animals.

The analysis described thus far reveals several significant age differences in spatial coding by CA1 and hilar hippocampal neurons that are task dependent. It was also of interest to compare within each age group the differences in spatial specificity, reliability, and MVL across the CA1 and hilar subpopulations of hippocampal cells and across behavioral tasks. Such an interest stems from previous experiments that have shown that hilar neurons recorded from young adult rats during spatial memory tests are associated with more specific place fields than CA1 neurons (e.g., Mizumori et al., 1989). The latter result suggests that the CA1 and dentate hilar regions differentially contribute to hippocampally mediated spatial processing. The following analysis determined whether such a distinction is also evident in aged animals. Furthermore, we evaluated in young and old animals whether such regional distinctions persist across different behavioral test situations. If they do not persist, this suggests that the manner in which animals use an environment contributes to the organization of neural representations of space in the hippocampus.

A two-way ANOVA was performed on the specificity scores of cells recorded from young animals. The factors of interest were the subpopulation of cells recorded (CA1 or hilus) and behavioral task (forced-choice or spatial memory). Hilar neurons exhibited significantly greater specificity than CA1 neurons, $F(1,219)=15.74, p<.001$, in both forced-choice and spatial memory situations. Furthermore, place specificity scores of cells recorded during the spatial memory task were signifcantly greater than the scores of cells recorded during the forced-choice task, $F(1,219)=5.19, p<.05$. No interaction effect was observed. Although no significant regional or task variations were observed for reliability scores, MVL values were significantly greater for hilar neurons than for CA1 neurons in both tasks, $F(1,219)=15.09, p<.001$. There was 

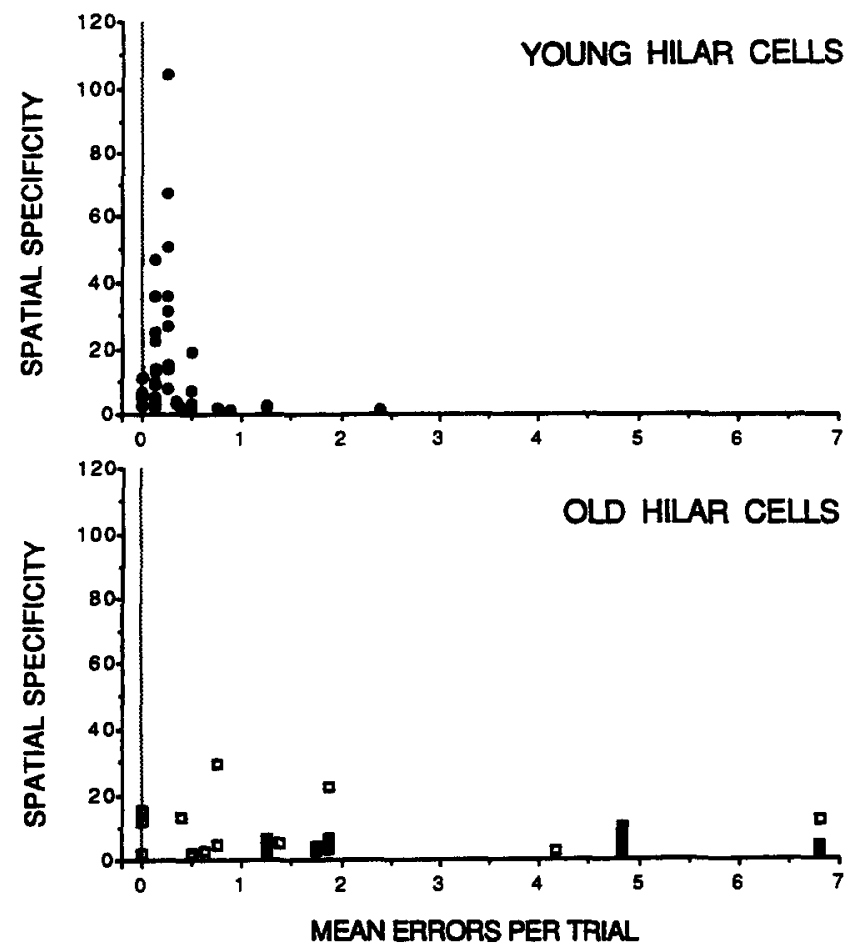

Figure 8. Scatter plot distribution of spatial specificity scores of hilar cells from young (top) and aged (bottom) rats as a function of the average number of errors made during the training session in which a given cell was recorded. When analyzed individually, no statistical correlation was found between choice accuracy and specificity score. However, when data from the two age groups were combined, a significant relationship between these variables emerged (see text).

no significant effect of task and no interaction effect for MVL. These data reveal that task demands importantly affect the specificity with which location information is coded by CA1 and hilar neurons.

Compared with the results presented for young rats, a different pattern of effects emerged for place cells of aged animals. A two-way ANOVA revealed a significant interaction

Table 1

Correlation Coefficients Relating Spatial Specificity and Reliability Scores

\begin{tabular}{llllll}
\hline Age & Cell type & $r$ & $p$ & $F$ & $d f$ \\
\hline \multicolumn{5}{c}{ Forced-choice training } \\
Young & CA1 CS & .36 & $<.01$ & 10.96 & 1,73 \\
Young & Hilar CS & .58 & $<.01$ & 13.42 & 1,28 \\
Old & CA1 CS & .04 & $n s^{*}$ & & \\
Old & Hilar CS & .32 & $n s^{* *}$ \\
\hline \multicolumn{5}{c}{ Spatial memory training } \\
Young & CA1 CS & .27 & $<.05$ \\
Young & Hilar CS & .54 & $<.001$ & 17.30 & 1,44 \\
Old & CA1 CS & .50 & $<.001$ & 15.60 & 1,48 \\
Old & Hilar CS & .44 & $<.01$ & 11.01 & 1,46 \\
\hline
\end{tabular}

Note. $\mathrm{CS}=$ complex spike.

${ }^{*} p>.10 .{ }^{* *} p<.07$.
Table 2

Correlation Coefficients Relating Spatial Specificity and Mean Vector Length Scores

\begin{tabular}{llcccc}
\hline Age & Cell type & $r$ & $p$ & $F$ & $d f$ \\
\hline \multicolumn{7}{c}{ Forced-choice training } \\
Young & CA1 CS & .37 & $<.010$ & 11.09 & 1,73 \\
Young & Hilar CS & .57 & $<.010$ & 12.29 & 1,28 \\
Old & CA1 CS & .57 & $<.001$ & 30.09 & 1,67 \\
Old & Hilar CS & .26 & $n s^{*}$ \\
\hline \multicolumn{7}{c}{ Spatial memory training } \\
Young & CA1 CS & .51 & $<.0001$ & \\
Young & Hilar CS & .60 & $<.0010$ & 23.69 & 1,73 \\
Old & CAl CS & .43 & $<.0100$ & 10.46 & 1,44 \\
Old & Hilar CS & .55 & $<.0010$ & 18.44 & 1,46 \\
\hline Note. CS $=$ complex spike. \\
${ }^{*} p>.10$.
\end{tabular}

effect of subpopulation of cells recorded and behavioral task for place specificity, $F(1,193)=4.51, p<.05$, and MVL scores, $F(1,193)=9.03, p<.01$. In particular, during forced-choice training, specificity and MVL values were higher for hilar neurons than CA1 neurons. During spatial memory testing, place specificity scores were lower for hilar neurons than CA1 neurons. A similar analysis of the reliability scores revealed that CA1 place fields were significantly more reliable than hilar cells, $F(1,193)=3.90, p<.05$, during the spatial memory task. Together, the pattern of effects obtained for the aged rats indicates that task requirements differentially affected specific aspects of spatial representation by different subpopulations of hippocampal cells.

\section{Discussion}

The present study evaluated (a) the relative contribution of age changes in rule-based and trial-unique processing to the spatial performance decline of aged animals and (b) whether changes in hippocampal neural representations of space might, at least in part, account for this navigational impairment. Different possibilities can be offered to account for the observed, statistically defined pattern of behavioral aging effects. Old rats apparently learn new rules of a spatial task as effectively as young rats because there was no age difference in the rate of learning in a new spatial environment. It can be reasoned, then, that the differential rate of new learning in a familiar environment is not likely to be due to an impaired ability to learn new rules per se. Moreover, an age-related deficiency in the ability to learn trial-unique information cannot explain the slower rate of learning when aged rats were required to change the manner in which they use (presumably) familiar environmental cues because a similar effect should have been observed when aged rats learned the same spatial task in a new environment. An alternative explanation is that the old rats are less flexible when it comes to adopting new rules imposed on old information. Age-related deficits in learning trial-unique information may instead account for the relatively poorer asymptotic performance of the old rats.

These behavioral data can be taken as evidence that age-related changes in at least the hippocampus and possibly 
also frontal cortex contribute to the spatial performance deficit. Specifically, age changes in hippocampal function may be responsible for the age difference in asymptotic performance on the memory task, and frontal cortical dysfunction may contribute to the apparently less flexible behavior exhibited by aged rats when they were required to apply new rules to old information. Alternatively or in addition, hippocampal dysfunction may influence the flexibility of strategy selection because rats with CA1 lesions exhibit an initial acquisition deficit when learning a spatial task in one environment but normal acquisition when the same animals then learn the same spatial task in a different environment (Mizumori, Garcia, Raja, \& Volpe, 1995).

This interpretation of the behavioral differences observed between young and aged rats is clearly speculative, especially because, although statistical significance was achieved, visual inspection of Figure 3 alone may not lead one to conclude that there are dramatic, qualitative age changes in the rates of learning in the two environmental conditions. It must be kept in mind that the difference between conditions could be more quantitative because the initial performance level differed for the aged animals during the two conditions. It is also worth noting that the age difference in choice accuracy at the end of the acquisition period is not as impressive as the statistically reliable age-related impairment shown in Figure 2. This could result from two factors: (a) Figure 2 includes behavioral data acquired during recording sessions, which occurred up to 2 months after acquisition; (b) we often observe that during extended asymptote spatial maze training young animals tended to make on average fewer errors as they become more expert on the task, whereas aged rats often make more errors. When combined, these two factors may have contributed to the more apparent impaired performance of aged rats during asymptote performance (see Figure 2).

Given that at least the hippocampus contributes to spatial performance deficits of aged rats, it was of interest to examine how age-related changes in the properties of hippocampal spatial representation play a role. Redistribution of hippocampal spatial coding was evident when old animals performed the spatial memory, and not the forced-choice, task. In particular, CA1 place cells of aged rats showed greater spatial specificity than those of young rats, whereas hilar place cells showed less specific coding. Similar patterns of change were also observed for directional coding and to a lesser extent reliability (see Figures 4 to 6 ). Spatial specificity scores were significantly correlated with reliability and MVL measures during spatial memory tests for both young and old rats (see Table 2). Thus, multiple measures suggest an age-related change in the organization of spatial coding in the hippocampus.

Understanding the functional significance of representational reorganization requires consideration of the possible contribution of CA1 and hilar regions to spatial learning. To begin to examine this issue, we evaluated within each age group the relationship between place field properties of cells recorded in different hippocampal subregions and performance on the maze. Consistent with previous reports (e.g., Mizumori et al., 1989), hilar place fields of young animals were more spatially selective than CA1 place fields when tested in either the forced-choice or spatial memory tasks. However, cells of both regions showed greater spatial selectivity during spatial memory tests than during forced-choice tests. This latter result suggests a relationship between the mnemonic demands of a task and place field properties in general: Place fields became more spatially selective under conditions in which the sensory environment remained constant (as it did in the same room condition) and the task requirements changed.

Although the relative contribution of the young hilus and CA1 to maze performance is not task dependent, each subregion may engage different neural integration of location information so that different contributions can be made to behavior. One possibility is that the apparent decline in spatial selectivity through successive stages of processing within hippocampus may reflect progressively enhanced association of afferent spatial information with (nonspatial) contextual information (Mizumori \& Williams, 1993). In this way, CA1 can come to code the general spatial context, or referent, in which specific (trial-unique) choices are made, and the dentate hilus may guide the selection of these individual choices (Mizumori et al., 1995).

Like young animals, hilar neurons of aged rats exhibited greater spatial selectivity than CA1 neurons during the forcedchoice task. Unlike young animals, however, hilar neurons showed much less spatially selective firing than CA1 neurons when aged animals performed the spatial memory task. It is postulated that in aged rats there is a deficiency in the dynamic and responsive neural process (which normally occurs in young animals) that is responsible for increased selectivity of hilar spatial representations with increased memory demands.

A previous study (Barnes et al., 1983) found that CA1 place cells of aged rats were less specific than those of young rats during forced-choice testing on an eight-arm radial maze. Their results stand in contrast to those of the present study. Careful consideration of differences in methodology do not provide an obvious explanation of the different results. Other possibilities include differences in cell sampling and differences in subpopulations of aged rats tested. Memory-impaired aged rats show clear alterations in hippocampal physiological function that are not apparent in memory-intact aged or young rats (e.g., Jiang, Owyang, Hong, \& Gallagher, 1989). The aged rats used in the present study were memory impaired, whereas the memory condition of Barnes et al.'s animals was not evaluated. Therefore, it is unclear whether direct comparison between results of this study and those of Barnes et al. (1983) is valid. Of relevance to the latter point, a more recent study from Barnes' laboratory (Markus, Barnes, McNaughton, Gladden, \& Skaggs, 1994) reported that, although not statistically significant, memory-impaired aged rats tend to exhibit CA1 place fields of greater spatial selectivity than young rats.

The results of this study are entirely consistent with data emerging from different laboratories that hippocampal spatial representations are contextually dependent and as such are very dynamic and responsive to environmental changes. The present study further shows that hippocampal spatial representations not only reflect current environmental conditions but also task demands. This form of dynamic response by hippocampal neurons is congruent with the generally held view that the hippocampus plays an important role in the mnemonic processing of environment-derived sensory information (e.g. 
McNaughton et al., 1996; McNaughton, Leonard, \& Chen, 1989). The present study further extends into the aging organism our appreciation of dynamic neural codes of hippocampus: Although specific and reliable hippocampal place fields are observed in aged rats performing in a familiar environment (also see Markus et al., 1994; Tanila, Shapiro, \& Eichenbaum, 1995), these cells may still contribute to suboptimal spatial learning by responding differently to changing task demands.

A fundamentally important issue that remains to be resolved before we fully understand not only aged, but also young adult, spatial learning concerns whether or not cells with smaller receptive fields necessarily code more specific information than cells with larger fields. If more broad place fields of CA1 reflect the association of spatial and nonspatial (e.g., contextual) information, and the very spatially restrictive hilar place fields represent only spatial location information, then it could be argued that although CA1 neural codes contain broadly integrated information, the code defines a specific context in which a location is relevant. In that sense, CA1 cells may represent more information than cells with smaller receptive fields.

With regard to our understanding of the neurobiology of age-related learning deficits, our finding of representational reorganization with age clearly indicates that, although essential, it is not sufficient to study the relationship between the structural or chemical integrity of the aging brain and behavior. In addition, one must be concerned with the dynamic nature of neurobiological systems and how reorganization of representations could support alternate behavioral strategies.

\section{References}

Barnes, C. A. (1979). Memory deficits associated with senescence: A neurophysiological and behavioral study in the rat. Joumal of Comparative and Physiological Psychology, 93, 74-104.

Barnes, C. A. (1990). Animal models of age-related cognitive decline. In F. Boller \& J. Grafman (Eds.), Handbook of neuropsychology (Vol. 4, pp. 169-196). New York: Elsevier.

Barnes, C. A., Green, E. J., Baldwin, J., \& Johnson, W. E. (1987). Behavioral and neurophysiological examples of functional sparing in senescent rat. Canadian Journal of Psychology, 41, 131-140.

Barnes, C. A., McNaughton, B. L., \& O'Keefe, J. (1983). Loss of place specificity in hippocampal complex-spike cells of the senescent rat. Neurobiology of Aging, 4, 113-119.

Fox, S. E., \& Ranck, J. B. Jr. (1975). Localization and anatomical identification of theta and complex-spike cells in the dorsal hippocampal formation of rats. Experimental Neurology, 49, 299-313.

Gallagher, M., Bostock, E., \& King, R. (1985). Effects of opiate antagonists on spatial memory in young and aged rats. Behavioral Neuroscience, 44, 374-385.

Jiang, H. K., Owyang, V., Hong, J. S., \& Gallagher, M. (1989). Elevated dynorphin in the hippocampal formation of aged rats: Relation to cognitive impairment on a spatial learning task. Proceedings of the National Academy of Sciences of the United States of America, 86, 2948-2951.

Lavoie, A. M. \& Mizumori, S. J. Y. (1994). Spatial, movement and reward-sensitive discharge by medial ventral striatum neurons of rats. Brain Research, 638, 157-168.

Lowry, A. M., Ingram, D. K., Olton, D. S., Waller, S. B., Reynolds, M. A., \& London, E. D. (1985). Discrimination learning requiring different memory components in rats: Age and neurochemical comparisons. Behavioral Neuroscience, 99, 638-651.

Markus, E. J., Barnes, C. A., McNaughton, B. L., Gladden, V. L., \& Skaggs, W. E. (1994). Spatial information content and reliability of hippocampal CA1 neurons: Effects of visual input. Hippocampus, 4, 410-421.

McNaughton, B. L., Barnes, C. A., Gerrard, J. L., Gothard, K., Jung, M. W., Knierim, J. J., Kudrimoti, H., Qin, Y., Skaggs, W. E., Suster, M., \& Weaver, K. L. (1996). Deciphering the hippocampal polyglot: The hippocampus as a path integration system. Joumal of Experimental Biology, 199, 173-185.

McNaughton, B. L., Barnes, C. A., \& O'Keefe, J. (1983). The contributions of position, direction and velocity to single unit activity in the hippocampus of freely-moving rats. Experimental Brain Research, 52, 41-49.

McNaughton, B. L., Leonard, B., \& Chen, L. (1989). Corticalhippocampal interactions and cognitive mapping: A hypothesis based on reintegration of the parietal and inferotemporal pathways for visual information. Psychobiology, 17, 230-235.

McNaughton, B. L., O’Keefe, J., \& Barnes, C. A. (1983). The stereotrode: A new technique for simultaneous isolation of several single units in the central nervous system from multiple unit records. Journal of Neuroscience Methods, 8, 391-397.

Mizumori, S. J. Y. (1994). Neural representations during spatial navigation. Current Directions in Psychological Science, 3, 1-5.

Mizumori, S. J. Y., Barnes, C. A., \& McNaughton, B. L. (1990). Behavioral correlates of theta-on and theta-off cells recorded from hippocampal formation of mature young and aged rats. Experimental Brain Research, 80, 365-373.

Mizumori, S. J. Y., Barnes, C. A., \& McNaughton, B. L. (1992). Differential effects of age on subpopulations of hippocampal theta cells. Neurobiology of Aging, 13, 673-679.

Mizumori, S. J. Y., Garcia, P. A., Raja, M. A., \& Volpe, B. T. (1995). Spatial- and locomotion-related neural representation in rat hippocampus following long-term survival from ischemia. Behavioral Neuroscience, 109, 1081-1094.

Mizumori, S. J. Y., McNaughton, B. L., Barnes, C. A., \& Fox, K. B. (1989). Preserved spatial coding in hippocampal CA1 pyramidal cells during reversible suppression of $\mathrm{CA} 3$ output: Evidence for pattern completion in hippocampus. Journal of Neuroscience, 9, 3915-3928.

Mizumori, S. J. Y., Perez, G. M., Alvarado, M. C., Barnes, C. A., \& McNaughton, B. L. (1990). Reversible inactivation of the medial septum differentially affects two forms of learning in rats. Brain Research, 528, 12-20.

Mizumori, S. J. Y., Ward, K. E., \& Lavoie, A. M. (1992). Medial septal modulation of entorhinal single unit activity in anesthetized and freely moving rats. Brain Research, 570, 188-197.

Mizumori, S. J. Y., \& Williams, J. D. (1993). Directionally selective mnemonic properties of neurons in the lateral dorsal nucleus of the thalamus of rats. Joumal of Neuroscience, 13, 4015-4028.

Muller, R. U., Kubie, J. L., Bostock, E. M., Taube, J. S., \& Quirk, G. J. (1991). Spatial firing correlates of neurons in the hippocampal formation of freely moving rats. In J. Paillard (Ed.), Brain and space (pp. 296-333). New York: Oxford University Press.

O'Keefe, J. (1976). Place units in the hippocampus of the freelymoving rat. Experimental Neurology, 51, 78-109.

O'Keefe, J., \& Dostrovsky, J. (1971). The hippocampus as a spatial map. Preliminary evidence from unit activity in the freely-moving rat. Brain Research, 34, 171-175.

O'Keefe, J., \& Speakman, A. (1987). Single unit activity in the rat hippocampus during a spatial memory task. Experimental Brain Research, 68, 1-27.

Olton, D. S., \& Samuelson, R. J. (1976). Remembrance of places 
passed: Spatial memory in rats. Journal of Experimental Psychology: Animal Behavior Processes, 2, 97-116.

Paxinos, G., \& Watson, C. (1986). The rat brain in stereotaxic coordinates. New York: Academic Press.

Ranck, J. B. Jr. (1973). Studies on single neurons in dorsal hippocampus formation and septum in unrestrained rats: Part I. Behavioral correlates and firing repertoires. Experimental Neurology, 41, 461555.

Rapp, P., Rosenberg, R., \& Gallagher, M. (1987). An evaluation of spatial information processing in aged rats. Behavioral Neuroscience, 101, 3-12.

Rose, G., Diamond, D., \& Lynch, G. S. (1983). Dentate granule cells in the rat hippocampal formation have the behavioral characteristics of theta neurons. Brain Research, 266, 29-37.
Tanila, H., Shapiro, M., \& Eichenbaum, H. (1995). Hippocampal place fields in aged rats with spatial memory deficit. Society for Neuroscience Abstracts, 21, 943.

Winocur, G. (1988). A neuropsychological analysis of memory loss with age. Neurobiology of Aging, 9, 487-494.

Winocur, G., \& Moscovitch, M. (1990). Hippocampal and prefrontal cortex contribution to learning and memory: Analysis of lesion and aging effects on maze learning in rats. Behavioral Neuroscience, 104, 544-551.

Received August 30, 1995

Revision received December 7, 1995

Accepted March 19, 1996 